\title{
MOLECULES IN GALAXIES: RESULTS FROM BELL LABORATORIES
}

\author{
A. A. Stark \\ AT\&T Bell Laboratories \\ Crawford Hill Laboratory; Holmdel, NJ 07733
}

\begin{abstract}
A decade of galaxy observations at the Crawford Hill $7 \mathrm{~m}$ antenna are summarized. Significant results include the mapping of spiral arms in $\mathrm{CO}$, the demonstration that gas in many spiral galaxies is roughly half atomic and haif molecular, the detection of extragalactic $\mathrm{HCO}^{+}, \mathrm{CS}^{13} \mathrm{CO}$ and $\mathrm{C}^{18} \mathrm{O}$, and the observation of molecular material in the halo of M82.
\end{abstract}

The $7 \mathrm{~m}$ diameter millimeter-wave antenna at AT\&T Bell Laboratories, Crawford Hill, has been used for a variety of extragalactic observational projects. This paper briefly summarizes the results.

Early observations concentrated on the feasibility of detections. HCN and $\mathrm{HCO}^{+}$were detected in M82 and NGC253 (Stark and Wolff 1979). CS was detected by Henkel and Bally (1985). ${ }^{13} \mathrm{CO}$ was detected in several galaxies (Encrenaz et al. 1979), showing that the ${ }^{12} \mathrm{CO} /{ }^{13} \mathrm{CO}$ ratio varied from galaxy to galaxy, but that typical values were higher than GMCs in the Milky Way. Stark and Carlson (1984) detected $\mathrm{C}^{18} \mathrm{O}$ in M82, at a level consistent with terrestrial isotopic abundances. Stark and Carlson detected $\mathrm{CO}$ emission from the filaments above and below the disk of M82. This molecular gas is in an unusual state, probably a result of galaxy collisions. Seyfert galaxies were detected in ${ }^{12} \mathrm{CO}$ (Bieging et al. 1981), but subsequent work (Blitz et al. 1986) showed that most Seyferts are not extraordinary in their CO brightness.

Many of the observations relate to the nature of spiral structure, and its appearance in the CO line. Stark et al. (1979), Stark (1979, 1984a), Boulanger et al. (1981), Ryden and Stark (1986), and Casoli et al. (1987) mapped spiral arms in M31, where the arm/interarm contrast in CO brightness exceeds 10. Ryden and Stark observed a sudden shift in velocity across the arm, interpreted by them as a dynamical effect of the spiral density wave; however, Casoli et al. argue that this is in fact an effect of the warp which is known from Hr line observations. Stark et al. (1987) observed total CO emissivity in a sample of 29 galaxies, some with well-defined spiral structure, and some without. They found that spiral structure does not greatly affect a galaxy's CO brightness, and 593

D. McNally (ed.), Highlights of Astronomy, Vol. 8, 593-594.

(c) 1989 by the IAU. 
interpreted this in a model where GMC formation is very efficient: if a spiral density wave is present, it organizes the formation of GMCs along the arms; if a spiral density wave is not present, then GMCs form throughout the disk anyway.

Recent work concentrates on the overall $\mathrm{CO}$ emissivity of galaxies. Verter (1983) showed that CO emissivity increases with luminosity class, and that Sb spirals seem to have the highest $\mathrm{CO}$ emissivity per unit mass, although this quantity has considerable unexplained scatter. Polk et al. (1988) modeled the overall CO emission from galaxies as the result of two populations of molecular clouds: giant molecular clouds and diffuse clouds. They showed that diffuse clouds can make a disproportionate contribution to the total emissivity. A sample of Virgo cluster spiral galaxies (Stark et al. 1986) has been analysed (Knapp et al. 1987) to show that the $\mathrm{CO}$ emissivity per $\mathrm{H}_{2}$ molecule is about the same in these galaxies as in the Milky Way, and that this sample of "normal" spiral galaxies contains some galaxies which are mostly molecular, and some galaxies which are mostly atomic. Richmond and Knapp (1986) found that NGC 4565 is approximately half atomic and half molecular, but the molecular component is more centrally concentrated. Total CO emission is well-correlated with far-IR emission, but Stark (1984b) showed that there is little molecular gas in the center of M31, even though IRAS shows more far-IR radiation from the center than from the spiral arms, which are $\mathrm{CO}$ bright.

\section{REFERENCES}

Bieging, J. H., Blitz, L., Lada, C. J., and Stark, A. A. 1981 Ap. J., 247, 443.

Blitz, L., Mathieu, R. D., and Bally, J. 1986 Ap. J., $311,142$.

Boulanger, F., Stark, A. A., and Combes, F. 1981 Astr. Ap., 93, L1.

Casoli, F., Combes, F., and Stark, A. A. 1987 Astr. Ap., 173, 43.

Encrenaz, P. J., Stark, A. A., Combes, F., and Wilson, R. W. 1979 Astr. Ap., 78, L1.

Henkel, C., and Bally, J. 1985 Astr. Ap., 150, L25.

Knapp, G. R., Helou, G. and Stark, A. A. 1987 A. J., 94, 54.

Polk, K. S., Knapp, G. R., Stark, A. A., and Wilson, R. W. 1988 Ap. J., 332, 432.

Richmond, M. W., and Knapp, G. R. 1986 A. J., 91, 517.

Ryden, B. S., and Stark, A. A. 1986 Ap. J., 305, 823.

Stark, A. A. 1979 Ph. D. thesis, Princeton University.

Stark, A. A. 1984a in The Milky Way Galaxy ed. H. Van Woerden et al., p.p. 445.

Stark, A. A. 1984b B.A.A.S 18, 538.

Stark, A. A., and Carlson, E. R. 1984 Ap. J., 279, 122.

Stark, A. A., Elmegreen, B. G., and Chance, D. 1987 Ap. J., 322, 64.

Stark, A. A., Frerking, M. A., and Linke, R. A. 1978 B.A.A.S. 11, 415.

Stark, A. A., Knapp, G. R., Bally, J., Wilson, R. W., Penzias, A. A., and Rowe, H. E. 1986 Ap. J., 310, 660 .

Stark, A. A. and Wolff, R. S. 1979 Ap. J, 229, 118.

Verter, F. 1983 Ph. D. Thesis, Princeton University. 